\title{
20 YEARS OF SOHO
}

I Bernhard Fleck ${ }^{1}$ and Daniel Müller ${ }^{2}$ - DOI: http://dx.doi.org/10.1051/epn/2016306

- ${ }^{1}$ ESA, SOHO Project Scientist and ${ }^{2}$ ESA, Solar Orbiter Project Scientist

The Solar and Heliospheric Observatory (SOHO), a joint mission of ESA and NASA, has provided unparalleled insight into the Sun over the past 20 years - from its interior, through the hot and dynamic atmosphere, out to the solar wind and its interaction with the interstellar medium. SOHO also plays a vital role in forecasting potentially dangerous space weather situations by continuously monitoring solar storms, and unexpectedly also became the most prolific discoverer of comets in the history of astronomy. 


\section{P27: Extreme- ultraviolet image of the Sun taken by $\mathrm{SOHO}$ on 14 September 1999.}

v FIG. 1: The changing face of the Sun and - the corona - from 1996 to 2015 over almost two complete solar cycles. The

images were taken by SOHO/EIT in the Fe XV 284 Å filter showing the Sun's hot corona at about 2 million ${ }^{\circ} \mathrm{C}$ and illustrate the waxing and waning of activity during the 11-year solar cycle. its outer atmosphere

\section{A cooperative effort between ESA and NASA}

The SOHO spacecraft was built for ESA by Europe's aerospace industry and was launched by NASA on 2 December 1995. Mission control is based at NASA's Goddard Space Flight Center near Washington DC. Of the satellite's 12 science instruments, nine come from multinational teams led by European scientists, and three from US-led teams.

SOHO is stationed 1.5 million $\mathrm{km}$ from the Earth. It follows a halo orbit around the $1^{\text {st }}$ Lagrangian point, where it enjoys an uninterrupted view of the Sun. There, the combined gravity of Earth and Sun keeps SOHO in an orbit locked to the Earth-Sun line. Originally planned for a two-year mission, its numerous extensions have allowed it to cover nearly two 11-year solar cycles. SOHO is thus the longest-lived Sun-watching mission, which by now allows us to compare the Sun's behaviour not only at different times in one cycle, but also during different cycles (FIG. 1).

\section{Seeing the inside of the Sun}

Just as seismology reveals the Earth's interior by studying earthquake waves, solar physicists probe the interior of the Sun using a technique called helioseismology. The oscillations detectable at the visible surface are due to sound waves reverberating through the Sun's inner layers. These oscillations are usually described in terms of normal modes. By precisely measuring the mode frequencies, one can infer the Sun's temperature, density, atomic abundances, interior structure, as well as the age of the solar system, and even pursue tests of the constancy of the gravitational constant.

In addition to exploiting the techniques of "global helioseismology", SOHO opened and pioneered the new field of "local area helioseismology", providing the first 3-D images and flow maps of the interior of a star, and even images of activity on the far side of the Sun.

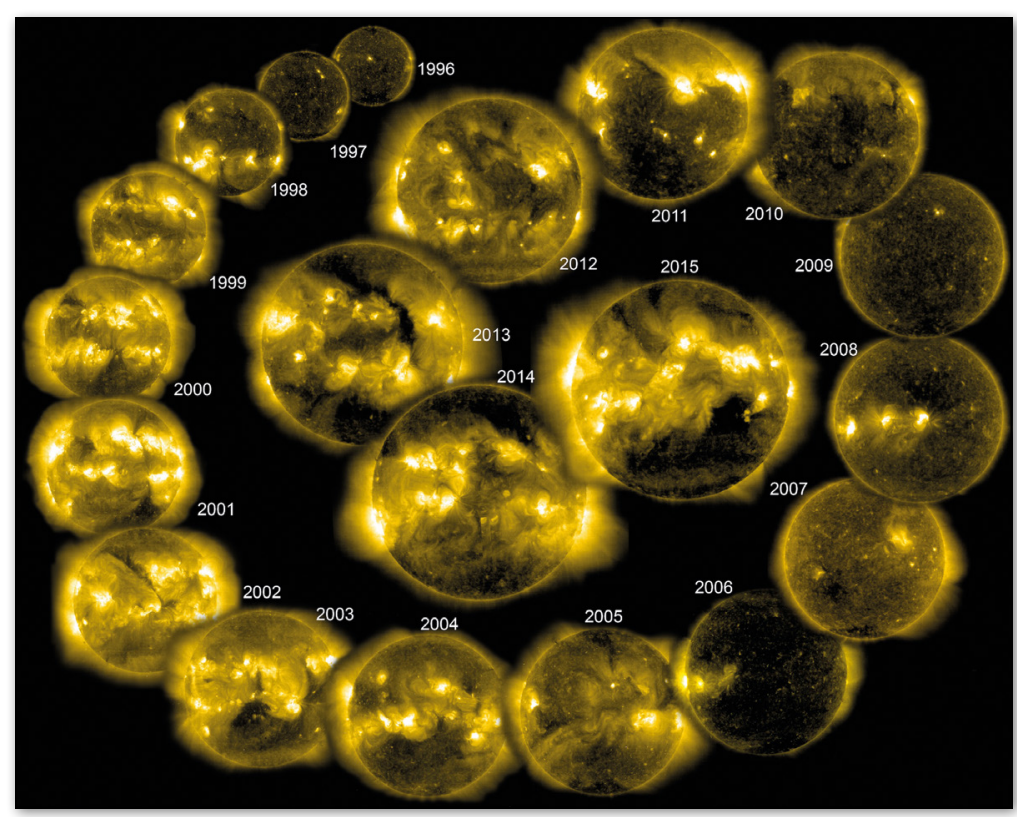

SOHO discovered 'sunquakes' and a slow subsurface current of gas flowing from the equator towards the poles. Deeper inside the Sun, about a third of the way towards the center at the transition between its turbulent outer shell - the convection zone - and the more orderly radiative zone, $\mathrm{SOHO}$ found that the Sun's rotation profile changes abruptly (FIG. 2). Near the equator the outer layers rotate faster than the inner layers. At mid-latitudes and near the poles, the situation is reversed. The boundary region between these two layers is of particular interest because it is where the solar dynamo that creates the Sun's ever-changing magnetic field is believed to operate.

SOHO also shed light on the 'solar neutrino problem': When $\mathrm{SOHO}$ was launched, there was a discrepancy between the rate at which neutrinos were predicted to be created by nuclear fusion in the deep solar interior and the rate measured at Earth. SOHO determined that the "standard model" of the solar interior was correct, and that the discrepancy had to be explained by the physics of the neutrino. This was subsequently confirmed by measurements of the Sudbury Neutrino Observatory, which provided compelling evidence for so-called flavour transitions of solar electron neutrinos, a discovery that was awarded the Nobel Prize in Physics in 2015.

\section{The dynamic solar atmosphere and the solar wind}

The question of why the Sun's outer atmosphere, the corona, has a temperature of 1-2 million degrees when the visible surface is 'only' about $5500^{\circ} \mathrm{C}$ has long been a mystery of solar physics. SOHO has revealed an extremely dynamic atmosphere where magnetic flux is constantly emerging from the Sun's interior and the energy supply through "braiding" of the large-scale coronal magnetic field by small-scale flux replacement is sufficient to heat the tenuous corona. Moreover, SOHO observations led to the discovery of new dynamic phenomena such as solar tornadoes and global coronal waves - disturbances associated with coronal mass ejections that can travel around the entire solar globe.

One of the prime goals of $\mathrm{SOHO}$ has been to observe where the solar wind - electrically charged atomic particles streaming from the Sun - is produced and how it is accelerated to beyond 3 million $\mathrm{km} / \mathrm{h}$. Scientists have made great strides in answering this fundamental question by analyzing data from SOHO. They measured the acceleration profiles of both the 'slow' and 'fast' solar wind and found, e.g., that the fast solar wind streams into interplanetary space by 'surfing' on waves produced by vibrating magnetic field lines. SOHO also revealed that heavy solar wind ions in coronal holes - darker, cooler and less dense areas of the Sun's corona where the Sun's open magnetic field reaching into space allows hot ionized gas to escape - flow faster and are heated hundreds of times more strongly than protons and electrons, and 


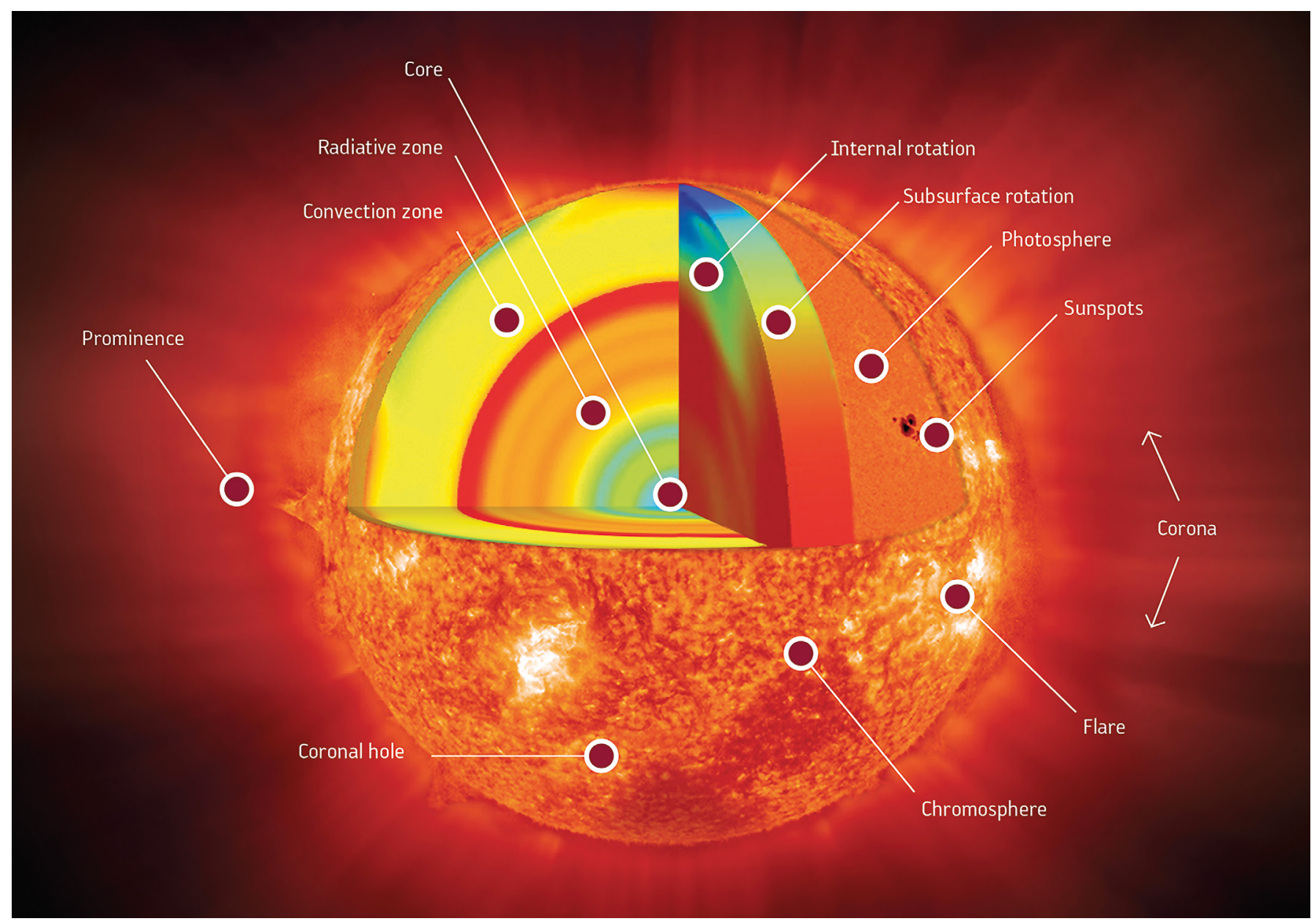

$\Delta$ FIG. 2: The anatomy of our Sun. Left cutaway: The Sun's interior explored with sound waves. Red depicts layers where sound travels faster than predicted by theory, implying that the temperature is higher than expected, while turquoise and green indicates slower speeds and lower temperatures. The prominent red layer marks the transition between the turbulent outer convection zone and the more stable inner radiative zone. Right cutaway: The Sun's internal rotation, where red depicts fast rotation and blue slower rotation. Outer layers: Visible light images show sunspots, cool dark features in the photosphere, which lies below the chromosphere. Flares, resulting from the release of a buildup of magnetic energy, and coronal mass ejections (CMEs, giant clouds of electrically charged atomic particles launched into space) often occur in magnetically active regions around sunspot groups.

that they have highly anisotropic temperatures reaching hundreds of millions degrees Kelvin in the direction perpendicular to the magnetic field.

\section{SOHO, the space weather watchdog}

With its near-continuous monitoring of the Sun, SOHO has revolutionized our understanding of the Sun-Earth connection and dramatically boosted space weather forecasting capabilities.

The major driver of space weather are coronal mass ejections (CMEs), the most powerful eruptions in the Solar System, which propel billions of tonnes of ionised gas into space at millions of kilometers per hour (FIG.3). If CMEs hit Earth, in addition to causing intense auroral displays in polar regions by electrically charging atoms in our upper atmosphere, they can cause major geomagnetic storms, damage satellites, disrupt telecommunications, endanger astronauts, lead to corroded oil pipelines and cause current surges in power lines. As our society becomes increasingly dependent on space-based technologies, our vulnerability to "space weather" becomes more obvious, and the need to understand it and mitigate its effects becomes more urgent.
$\mathrm{SOHO}$ is a pioneer in detecting when such a solar storm is incoming. It has studied more than $20000 \mathrm{CMEs}$ to date, pinpointing their sources on the Earth-facing hemisphere of the Sun, and determining their speed and direction to provide up to three days' warning - sufficient to take mitigating action on Earth. From its vantage point matching Earth's orbit, the observatory also makes in situ measurements when a CME and its energetic particles arrive.

\section{The day star's brightness variations}

Monitoring variations of the total solar irradiance (TSI) as well as variations in the extreme ultraviolet (EUV) flux from the Sun are an important part of SOHO's long-term studies. Both these quantities are important for understanding possible effects of solar variability on climate and for disentangling natural variations from anthropogenic effects. While the total solar irradiance varies by less than $0.1 \%$ over an 11 -year solar cycle, the irradiance in the EUV part of the spectrum changes by as much as $30 \%$ on the time scale of weeks and by a (wavelength-dependent) factor of 2 to 100 over the solar cycle. 


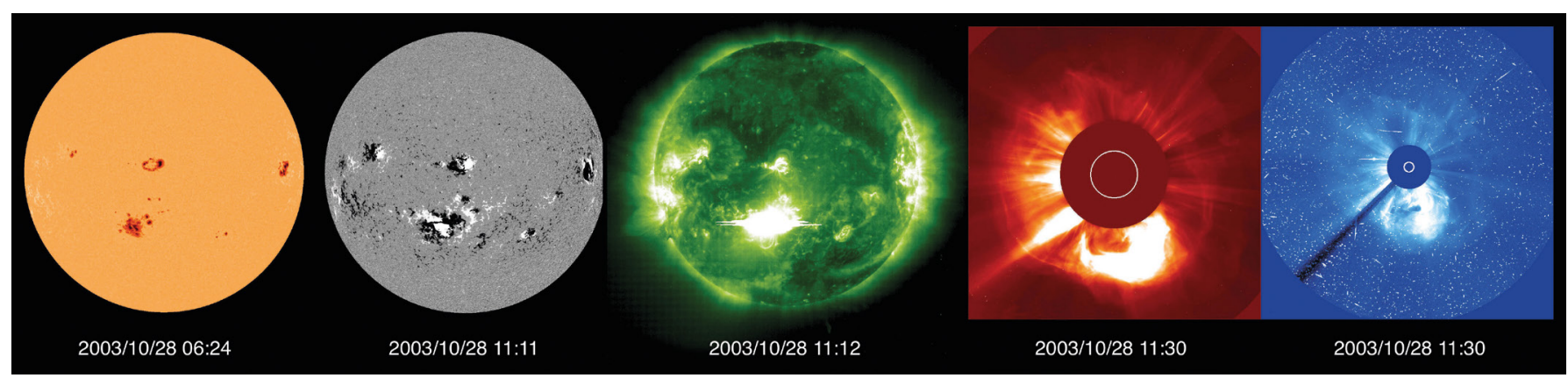

\FIG. 3: Halloween Storms of 2003: For two weeks in 0ctober-November 2003, the Sun featured three unusually large sunspot groups, which gave rise to 11 X-class flares - the most energetic class of flares - including the strongest ever recorded. First left: giant sunspot groups seen by SOHO's MDI instrument on 28 0ctober 2003 in visible light. Second: MDI magnetogram on the same day, illustrating the magnetic complexity of these active regions. Third: SOHO/EIT image at the time of an X-ray flare, seen as the bright emission just below the centre of the disc. The linear horizontal feature is an artefact due to saturation of the CCD detector. Fourth: SOHO/LASCO C2 image at minutes after the flare, with a 'halo CME' (Coronal Mass Ejection) completely surrounding the occulting disc. Fifth: LASCO C3 image of the expanding halo CME, where energetic particles hitting the CCD detector appear like 'snow'. The flare location and the halo were a clear indication that the CME was heading towards Earth.

\section{A prolific comet-hunter}

Besides watching the Sun, $\mathrm{SOHO}$ is also the most prolific comet discoverer of all time: more than 3000 comets have been found, over $95 \%$ of those by amateurs accessing SOHO's publicly available real-time data via the Internet. Prior to the launch of SOHO in 1995, only a dozen or so comets had ever been discovered from space, while some 900 had been discovered from the ground. Most of the comets discovered by SOHO are 'sungrazing' comets that perish in the Sun's heat. Some survive, albeit in various states of degradation: SOHO has watched many comets lose their heads and tails during their solar encounter (FIG. 4).

v FIG. 4: The demise of Comet ISON as it came within 1.2 million km of the Sun on 28 November 2013, fading from view in the following days. The small white circle in the center indicates the position and size of the Sun behind the telescope's occulting 'coronagraph mask', seen as the wider disc. The mask blocks out the dazzling light that otherwise drowns out this region, allowing details of the corona to be seen.

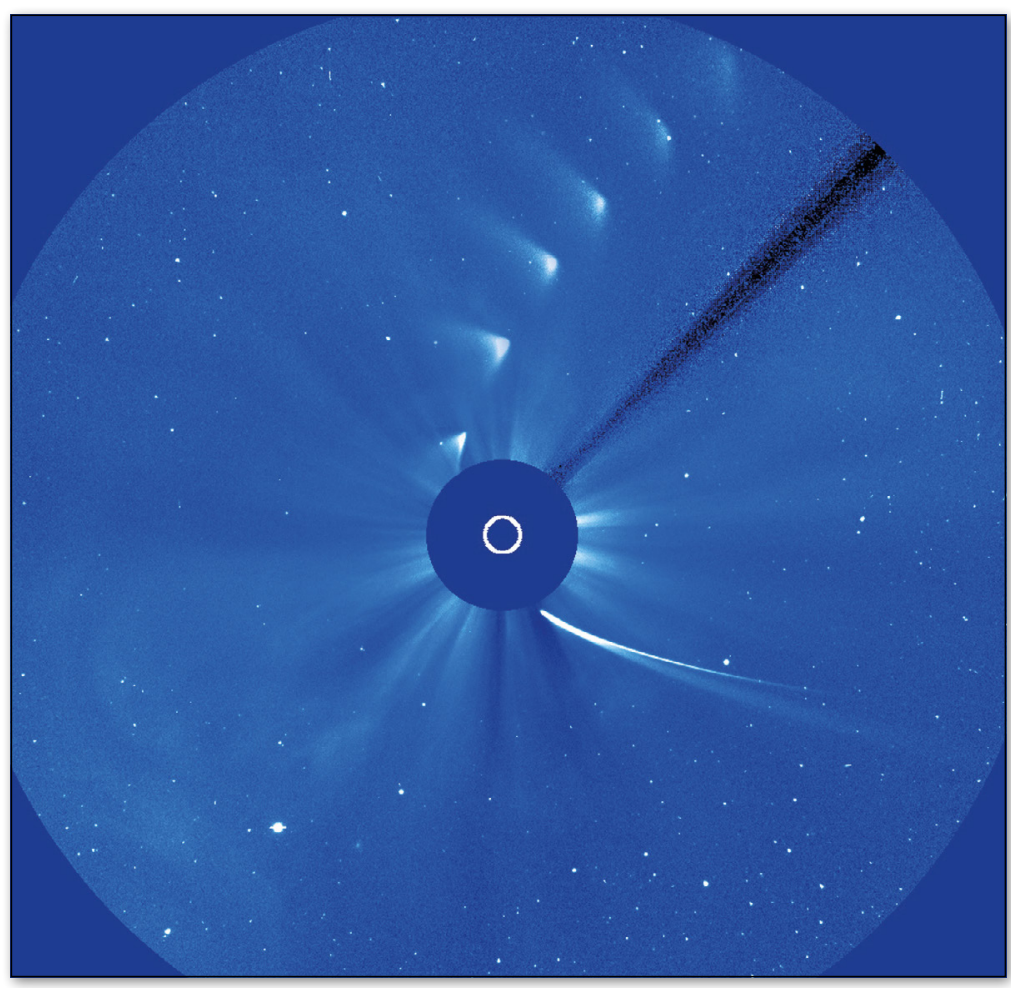

\section{Near-loss and dramatic recovery}

The SOHO mission almost ended on 25 June 1998 when control was lost during a routine spacecraft manoeuvre. It took three months to restore operations in one of the most dramatic recovery operations in space history, including just over two weeks to thaw frozen hydrazine propellant in the tank and pipes. Unexpectedly, all 12 instruments survived despite the extreme temperatures they suffered during the time that contact was lost.

But the drama was not over yet: Subsequently, all three gyroscopes of the spacecraft failed, the last one in December 1998. In a race against time, new software that no longer relied on gyroscopes for attitude control was developed and installed in February 1999, allowing the spacecraft to return to full scientific operations. This made SOHO the first spacecraft to be stabilised in three axes without gyroscopes. Despite these problems, engineers have kept $\mathrm{SOHO}$ functioning ever since, with all its instruments performing well.

\section{Conclusions and future}

In complex areas of research such as solar physics, progress is not made by just a few people. The scientific achievements of the SOHO mission are the results of a concerted, multi-disciplinary effort by a large international community of solar scientists, involving sound investment in space hardware coupled with a vigorous and well-coordinated scientific operation and interpretation effort.

Since 1995, SOHO has been joined by several other solar space missions, which have been providing observations at higher spatial and temporal resolution, from additional vantage points. However, even after 20 years and although only 8 of the original 12 science instruments are in active use, $\mathrm{SOHO}$ continues to provide unique and important measurements of our star.

Looking ahead, the next solar space missions are the joint ESA-NASA Solar Orbiter and the NASA Solar 
Probe Plus missions, both to be launched in 2018. Solar Orbiter will be leaving the plane of the ecliptic to explore the uncharted polar regions of the Sun, as well as investigate the connections between the Sun and its surrounding heliosphere from inside planet Mercury's orbit. Solar Probe Plus, on the other hand, will be approaching the Sun even further - to within 10 solar radii - to sample the plasma of the hot and tenuous solar corona in situ.

\section{Acknowledgements}

The great success of the $\mathrm{SOHO}$ mission is a tribute to the many people - too many to name here - who designed and built this exquisite spacecraft and these excellent instruments, to the engineers who brought it back from the dead (twice), and to the many people who diligently work behind the scenes to keep it up and running.

\section{About the Authors}

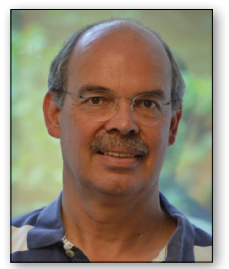

Bernhard Fleck is the ESA Project Scientist of the SOHO mission and has been based at NASA GSFC near Washington, DC, USA, since 1995. His research interests include the dynamics of the solar atmosphere, in particular wave-propagation characteristics in the chromosphere.

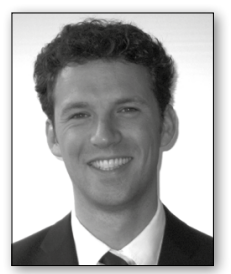

Daniel Müller worked from 2005-2010 as ESA's Deputy Project Scientist for SOHO at NASA GSFC. He is currently Project Scientist of the ESA-NASA Solar Orbiter mission, based at ESA's Space Research and Technology Centre (ESTEC) in the Netherlands. His research interests include the dynamics of the solar atmosphere, high-performance computing, and visualization techniques for complex data sets.

\section{Further reading}

[1] B. Fleck, V. Domingo, A.I. Poland, The SOHO Mission, Solar Phys. Vol. 162, No. 1-2 (1995)

[2] B. Fleck, P. Brekke, S. Haugan, L. Sánchez Duarte, V. Domingo, J.B. Gurman, A.I. Poland, Four Years of SOHO Discoveries Some Highlights, ESA Bulletin 102, 68 (2000):

http://soho.nascom.nasa.gov/publications/ESA_Bull102.pdf

[3] B. Fleck, Eight Years of SOHO: Some Highlights, ASSL 320, 139 (2005) http://adsabs.harvard.edu/abs/2005ASSL..320..139F

[4] B. Fleck, D. Müller, S. Haugan, L. Sánchez Duarte, T. Siili, J.B. Gurman, : Ten Years of SOHO, ESA Bulletin 126, 24 (2006): http://soho.nascom.nasa.gov/publications/ESA_Bull126.pdf

[5] F. Vandenbussche, SOHO's Recovery - An Unprecedented Success Story, ESA Bulletin 97, 39 (1999): http://soho.nascom. nasa.gov/operations/Recovery/vandenbu.pdf SOHO Web Site: http://soho.nascom.nasa.gov/ and http://soho.esac.esa.int 